\title{
Correlation Electron Cyclotron Emission Diagnostic and Improved Calculation of Turbulent Temperature Fluctuation Levels on ASDEX Upgrade
}

\author{
A.J. Creely, ${ }^{1,}$ | S.J. Freethy, ${ }^{1,2}$ W.M. Burke, ${ }^{1}$ G.D. Conway ${ }^{2}$ \\ R. Leccacorvi, ${ }^{1}$ W.C. Parkin, ${ }^{1}$ D.R. Terry,${ }^{1}$ and A.E. White ${ }^{1}$ \\ ${ }^{1}$ MIT Plasma Science and Fusion Center, Cambridge, MA, USA. \\ ${ }^{2}$ Max Planck Institute for Plasma Physics, Garching, Germany.
}

(Dated: April 5, 2018)

\begin{abstract}
A newly upgraded correlation electron cyclotron emission (CECE) diagnostic has been installed on the ASDEX Upgrade tokamak and has begun to make experimental measurements of electron temperature fluctuations. CECE diagnostics measure small amplitude electron temperature fluctuations by correlating closely spaced heterodyne radiometer channels. This upgrade expanded the system from six channels to thirty, allowing simultaneous measurement of fluctuation level radial profiles without repeat discharges, as well as opening up the possibility of measuring radial turbulent correlation lengths. Newly refined statistical techniques have been developed in order to accurately analyze the fluctuation data collected from the CECE system. This paper presents the hardware upgrades for this system and the analysis techniques used to interpret the raw data, as well as measurements of fluctuation spectra and fluctuation level radial profiles.
\end{abstract}

\section{INTRODUCTION}

Understanding and predicting plasma transport in the core of fusion plasmas will be vital to the design and operation of future devices. It is believed that in the core of many fusion plasmas, heat, particle, and momentum transport is driven dominantly by microinstabilities and plasma turbulence, which often far exceed transport levels from neoclassical effects [1. This turbulence can be measured as small amplitude fluctuations in the plasma temperature, density, potential, and magnetic field. Measurement of these fluctuations can shed light on the character of this turbulence and the role that it plays in plasma transport.

In addition to interest in the fundamental nature of the fluctuations on a purely experimental level, the continuing development of more advanced gyrokinetic simulations motivates measurement of turbulence as part of code validation efforts. While many studies focus on comparing experimental and simulated heat fluxes, recently it has become increasingly common to compare other experimental quantities to simulation outputs, such as temperature and density fluctuation amplitudes, spectra, and correlation lengths [2]. See Refs. [3], 44 and [5] for just a few recent studies comparing gyrokinetic codes to experimental measurements of turbulent fluctuations. It has also been suggested that these comparisons may be more constraining than heat flux comparisons, as they directly compare more fundamental turbulent phenomena [2].

One method of measuring such fluctuations is known as correlation electron cyclotron emission (CECE). CECE measures local, long wavelength $\left(k_{\theta} \rho_{s}<0.3\right.$, where $\rho_{s}$ is the ion Larmor radius evaluated at the sound speed), electron temperature fluctuations. Due to the thermal

\footnotetext{
* acreely@mit.edu
}

noise inherent in traditional ECE measurements, a single channel radiometer is typically unable to resolve turbulent temperature fluctuations above the noise floor. By correlating two closely spaced (radially) radiometer channels, however, one is able to eliminate uncorrelated thermal noise while retaining the turbulent fluctuation signal (assuming the structure is larger than the channel spacing). This technique has been utilized on a variety of machines worldwide 6 17.

This paper presents the design of and preliminary results from a newly upgraded CECE radiometer installed on the ASDEX Upgrade (AUG) tokamak, including the analysis techniques used to obtain these results. This diagnostic has already measured electron temperature fluctuation spectra with high sensitivity as well as finely resolved radial fluctuation profiles. The ability to simultaneously measure wide radial profiles of fluctuation levels as well as radial correlation lengths in a single plasma discharge makes this diagnostic a significant improvement over previous CECE systems.

\section{CECE DIAGNOSTIC}

The original CECE system on AUG is described in Ref. 15. The system detects second harmonic X-mode electron cyclotron emission and consists of waveguide optics, a radio frequency ( $\mathrm{RF}$ ) section, and an intermediate frequency (IF) section. A schematic block diagram is shown in Figure 1. The hardware section of this paper will focus primarily on an entirely new IF section, which upgraded the system from six to thirty channels, including six tunable frequency channels. This upgrade enables a far wider range of measurements, including finely resolved radial profiles, and has opened up the possibility of measuring radial correlation lengths of electron temperature fluctuations. In addition, the new hardware utilizes an optimized physical layout and improved ground isola- 


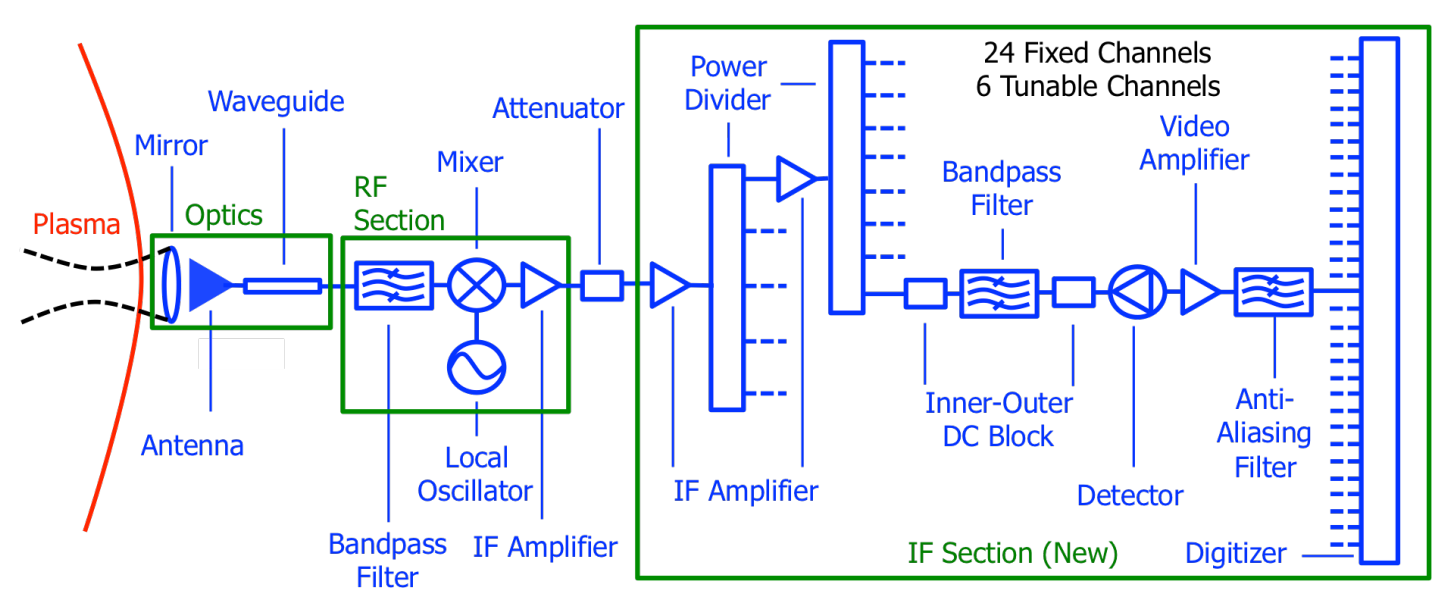

FIG. 1: Schematic block diagram of the new ASDEX Upgrade CECE system. There are twenty four fixed frequency bandpass channels and six tunable bandpass channels, for a total of thirty channels.

tion in order to reduce electronics noise in the system.

The AUG CECE system utilizes optics from the steerable Doppler reflectometer [18, which includes a steerable elliptical mirror, a smooth-bore Gaussian-beam antenna, an oversized waveguide, two tapers, and a fundamental waveguide [15]. Interchangeable sideband filters allow measurement frequencies of either 105 to $113 \mathrm{GHz}$ or 117 to $125 \mathrm{GHz}$. As an example, with an on-axis field of $2.5 \mathrm{~T}$, this corresponds to a radial range of $\rho_{\text {tor }}=0.4$ to 1.0 on ASDEX Upgrade, where $\rho_{\text {tor }}$ is the square root of the normalized toroidal magnetic flux. This RF section can also be interchanged to access other frequency ranges. This signal is then mixed down to intermediate frequencies within the RF section, with an output of 2 to $14 \mathrm{GHz}$. The signal then enters the new IF section.

The new IF section consists of six separate chassis. The first contains a low noise $30 \mathrm{~dB} 2-18 \mathrm{GHz}$ amplifier, before dividing the signal into six in a power divider, one for each of the five filter bank chassis (one signal is currently unused). Each of these signals is then amplified again by the same type of amplifier.

The divided signals are then sent to three fixed frequency filter bank chassis and two tunable filter bank chassis. Each fixed frequency chassis contains another eight-way power divider, and then eight cavity bandpass filters. These filter are interchangeable, but in the standard configuration the system operates with the $100 \mathrm{MHz}$ bandwidth filters spaced by $125 \mathrm{MHz}$ and $200 \mathrm{MHz}$ bandwidth filters spaced by $250 \mathrm{MHz}$. These two sets of filters cover a range from 4 to $8 \mathrm{GHz}$, and allow for radial profile measurements with fine radial resolution. Inclusion of two different bandwidths allows for comparison of the trade-offs between the two, as well as consistency checks of fluctuation measurements on independent channel sets.

After bandpass filtering, the signals pass through Schottky diode detectors and then are amplified again in $0-6.5 \mathrm{MHz}$ video amplifiers. The signals are then lowpass filtered at $1 \mathrm{MHz}$ to avoid aliasing in the digitizer.
Finally, the signals are digitized in a pair of synchronized 16 channel digitizers, typically at 4 mega samples per second.

The tunable frequency chassis are identical except that instead of fixed frequency cavity bandpass filters, they contain Yttrium Iron Garnet (YIG) tunable bandpass filters. These filters have a $200 \mathrm{MHz}$ bandwidth, and center frequencies that are tunable from $6 \mathrm{GHz}$ to 14 GHz. One of the tunable chassis has four channels, and the other has two (with space for two additional channels in a future upgrade).

All together, this system has thirty separate channels (twenty four fixed frequency and six tunable) in six modular chassis (one amplifier/divider chassis, three fixed frequency chassis, and two YIG chassis). As mentioned above, the physical layout within each chassis was optimized to reduce cable length and superfluous adapters in order to minimize electronics noise and loss. This design work produced the added benefit of making the system relatively compact despite the large number of channels. Finally, the new system contains greatly improved ground isolation throughout the six chassis, further reducing electronics noise. In particular, care was taken to maintain separate chassis and signal grounds among the six chassis, to prevent power supply noise and noise from surrounding diagnostics from entering the signal.

These improvements have enabled high quality, high resolution temperature fluctuation measurements, which will be shown in Section [IV] after Section III] describes the methods of analyzing the raw CECE data.

\section{DATA ANALYSIS TECHNIQUES}

This section describes new analysis techniques used to calculate the fluctuation spectra and total fluctuation amplitudes from the CECE diagnostic and then compares these techniques to previous techniques [6-17] using synthetic and experimental data. The method pre- 
sented here does not require any sort of absolute or crosscalibration of radiometer channels, thus eliminating the possibility of calibration-induced error. First, all of the equations used in the calculation of CECE temperature fluctuation spectra and integrated fluctuation levels are presented. Aspects of this methodology that differ from some past work are then addressed using the results of synthetic data testing. Finally, a method of subtracting coherent background noise from real data is presented.

\section{A. General Principles}

Fluctuation levels are extracted from the raw CECE diagnostic data using statistical time history analysis techniques outlined in References 19 21. As is derived in Appendix A, the total temperature fluctuation level measured by the CECE diagnostic is calculated as:

$$
\frac{\widetilde{T}}{T}=\sqrt{\frac{2}{B_{I F}} \int_{f_{1}}^{f_{2}} \frac{\operatorname{Re}\left\{\gamma_{c}(f)-\gamma_{b g}\right\}}{1-\operatorname{Re}\left\{\gamma_{c}(f)-\gamma_{b g}\right\}} d f}
$$

where $B_{I F}$ is the intermediate frequency bandwidth (the bandwidth of the bandpass filters used in the CECE system), $f_{1}$ and $f_{2}$ define the frequency range over which to integrate the coherence (the frequency width of the turbulent feature), $\gamma_{c}$ is the complex coherence function, and $\gamma_{b g}$ is the background coherence.

The complex coherence function, $\gamma_{c}$, is defined as (see References [19, 22, 27] for this definition, as well as References [20, 28, for an equivalent definition):

$$
\gamma_{c}(f)=\frac{G_{x y}(f)}{\sqrt{G_{x x}(f) G_{y y}(f)}}
$$

where $G_{x y}$ is the one-sided cross-spectral density function between channels $x$ and $y$, and $G_{i i}$ is the autospectral density function of channel $i$, defined as:

$$
G_{i j}(f)=2 F_{i}^{*}(f) F_{j}(f)
$$

where $F_{i}$ is the Fourier transform (frequency spectrum) of channel $i$ calculated with a $50 \%$ overlapping Hanning window ensemble averaged fast Fourier transform (FFT).

The background coherence, which will be discussed further in Section IIIC, is calculated as:

$$
\gamma_{b g}=\operatorname{Mean}\left[\gamma_{c}\right]_{f_{3}}^{f_{4}}
$$

where $f_{3}$ and $f_{4}$ define a frequency range far above the turbulent signal.

The uncertainty on the complex coherence function is calculated as the standard deviation, as described in [21]:

$$
\sigma_{\gamma_{c}}(f)=\sqrt{\frac{1}{2 n_{d}}\left(1-\left|\gamma_{c}(f)\right|^{2}\right)^{2}}
$$

where $n_{d}$ is the number of independent ensemble averaging windows.

This paper defines the sensitivity limit of the complex coherence function as two standard deviations above zero. In other words, a complex coherence that falls within two standard deviations of zero is considered to be indistinguishable from noise.

One can also propagate the uncertainty on $\gamma_{c}$ through Equation 1 to obtain the approximate uncertainty in $\widetilde{T} / T$, assuming that $\gamma_{c}$ is small compared to 1 (higher order terms are ignored):

$$
\sigma_{\widetilde{T} / T} \approx \frac{1}{\widetilde{T} / T} \frac{1}{2 B_{I F}} \sqrt{\sum_{i}\left(\sigma_{\gamma_{i}} \delta f\right)^{2}}
$$

where $\delta f$ is the frequency resolution of the Fourier transform. This equation is expressed as a finite sum, rather than an integral, to align most closely to how it is calculated in practice.

Finally, one can obtain the sensitivity limit of the integrated fluctuation level by appropriately integrating the standard deviation of the complex coherence function, given by Equation 5 , over the signal bandwidth. For the purposes of determining the sensitivity limit, the standard deviation of a signal with no coherence is used. This integration results in the following equation:

$$
\left.\frac{\widetilde{T}}{T}\right|_{\text {limit }} ^{\text {stat }}>\sqrt{\frac{2}{\sqrt{N}} \frac{B_{\text {sig }}}{B_{I F}} \sqrt{\frac{B_{\text {samp }}}{2 \cdot B_{\text {sig }}}}}
$$

where $N$ is the total number of data points, $B_{s i g}=$ $f_{2}-f_{1}$ is the signal bandwidth, $B_{\text {samp }}$ is the total sampling rate, and $B_{I F}$ is again the intermediate frequency bandwidth.

When $B_{s i g}$ is increased to the Nyquist Frequency, $B_{s i g}=(1 / 2) B_{\text {samp }}$, Equation 7 reduces to the ideal radiometer limit [8, 29]:

$$
\left.\frac{\widetilde{T}}{T}\right|_{\text {limit }} ^{\text {rad }}>\sqrt{\frac{2}{\sqrt{N}} \frac{B_{\text {sig }}}{B_{I F}}}
$$

where all variables are defined as above. This result is consistent with suggestions in References [10] and 30. that the ideal radiometer limit must be corrected when the sampling rate is significantly higher than the signal bandwidth.

This completes the set of equations required to analyze CECE fluctuation data.

The authors note that the equations presented here differ from past literature [5, 6, 8, 11, 13] in a few ways. Most of this past literature has calculated the total fluctuation level as:

$$
\frac{\widetilde{T}}{T}=\sqrt{\frac{2}{B_{I F}} \int_{f_{1}}^{f_{2}} \gamma_{x y}(f) d f}
$$


where all quantities are defined as before, and $\gamma_{x y}$ is the magnitude of the coherence function (see page 147 of Ref. 20], as well as Ref. [21]):

$$
\gamma_{x y}=\left|\gamma_{c}\right|
$$

Equation 1, used in this study, thus differs from Equation 9, used in the past, in three key ways:

1. The use of $\gamma_{c}$ as opposed to $\gamma_{x y}$.

2. The use of $\gamma /(1-\gamma)$ as opposed to just $\gamma$ in the integrand.

3. The subtraction of the background, $\gamma_{b g}$.

Each of these three differences is addressed in the derivation in Appendix A. In addition, Sections III B and III C compare the results of using Equation 1, as opposed to Equation 9, on both synthetic and real experimental data. Items 1 and 2 will be addressed in Section IIIB. which presents the results of synthetic data testing. Item 3 will be addressed in Section IIIC, which discusses why and how background subtraction is accomplished.

\section{B. Synthetic Data Testing}

This subsection will present the results of synthetic data testing to highlight the advantages of using $\operatorname{Re}\left\{\gamma_{c}\right\} /\left(1-\operatorname{Re}\left\{\gamma_{c}\right\}\right)$ as the integrand in Equation 11, assuming for now that $\gamma_{b g}=0$.

Synthetic data consists of two, zero mean, random gaussian signals, each consisting of 1000 bins of 1024 points, equivalent to roughly one second of data collected at $1 \mathrm{MHz}$ (assuming no bin overlap). A common broadband feature of varying bandwidths (between 20 and 300 $\mathrm{kHz}$ ), generated by low-pass filtering a random gaussian signal at the appropriate frequency, is then adjusted to be $1 \%$ of the total signal amplitude and is added to both signals. This set of data simulates two CECE channels, each with some uncorrelated background signal and a common $1 \%$ fluctuation of some given bandwidth. The common signal bandwidth is scanned in order to capture the effects of two possible issues (one at small bandwidth and another at large bandwidth) with integrating only $\gamma_{x y}$, as discussed below.

The synthetic data was analyzed using the techniques describe in Section III A. Specifically, this data was processed using variants of Equations 1 and 9 with different integrands, assuming that there is no coherent background $\left(\gamma_{b g}=0\right)$. Four integrands are tested: $\gamma_{x y}$, $\gamma_{x y} /\left(1-\gamma_{x y}\right), \operatorname{Re}\left\{\gamma_{c}\right\}$, and $\operatorname{Re}\left\{\gamma_{c}\right\} /\left(1-\operatorname{Re}\left\{\gamma_{c}\right\}\right)$.

The integration bounds used in Equation 1 were adjusted along with the bandwidth of the common broadband feature so as to capture the entire signal without integrating to significantly higher frequencies. In the results shown here, the $B_{I F}$ used in the synthetic data calculations was chosen to be $200 \mathrm{MHz}$, though a choice

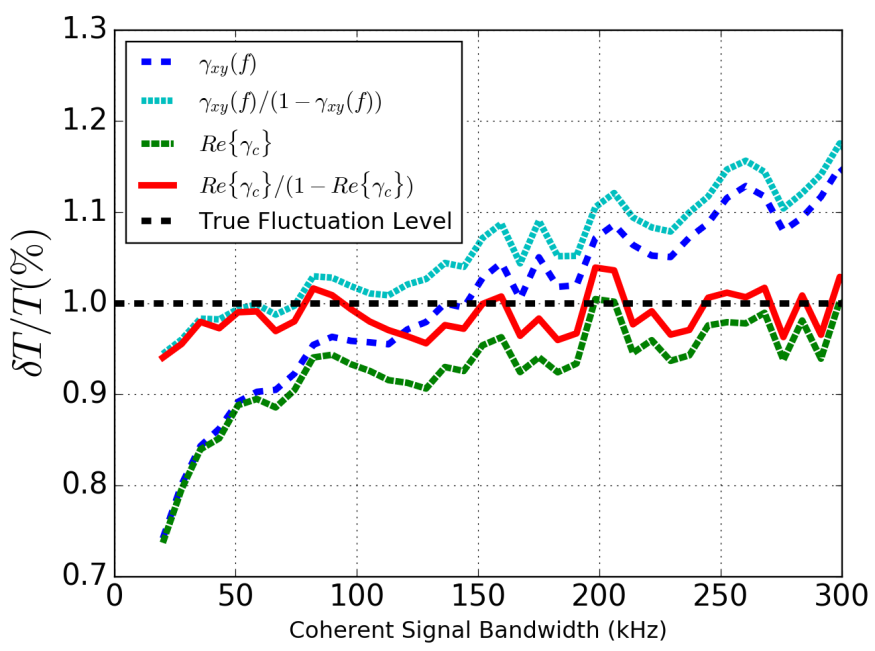

FIG. 2: Total fluctuation level of synthetic data calculated with various integrands in Equation 1. The

true fluctuation level of $1 \%$ is represented by the horizontal black dashed line. Integrals of $\gamma_{x y}$ (blue), $\gamma_{x y} /\left(1-\gamma_{x y}\right)$ (cyan), $\operatorname{Re}\left\{\gamma_{c}\right\}$ (green), and $\operatorname{Re}\left\{\gamma_{c}\right\} /\left(1-\operatorname{Re}\left\{\gamma_{c}\right\}\right)$ (red) are tested.

$\operatorname{Re}\left\{\gamma_{c}\right\} /\left(1-\operatorname{Re}\left\{\gamma_{c}\right\}\right)$ most accurately recovers the true fluctuation level over the widest range of common signal bandwidths.

of $100 \mathrm{MHz}$ was also tested and does not alter the conclusions drawn.

The results of this synthetic data analysis are shown in Figure 2. Ideally the analysis technique would recover the true fluctuation level of $1 \%$, shown as the horizontal black dotted line, at all fluctuation bandwidths. This synthetic data leads to two primary conclusions. First, the data shows that utilizing either $\gamma_{x y}$ or $\gamma_{x y} /\left(1-\gamma_{x y}\right)$ tends to increasingly overestimate the fluctuation level at large signal bandwidths. This is due to the continued integration of noise in $\gamma_{x y}$, which will always have a nonzero positive mean, and is proportionally larger as one considers larger and larger bandwidths. Since $\operatorname{Re}\left\{\gamma_{c}\right\}$ properly accounts for the phase between the two signals, however, it can have negative values and will not accumulate noise during integration.

Second, the data shows that utilizing either $\gamma_{x y}$ or $R e\left\{\gamma_{c}\right\}$ tends to underestimate the total fluctuation level at small signal bandwidths. This is due to the fact that at small bandwidths, the added common broadband signal is strong enough that it becomes significant in the autospectral density functions, $G_{i i}$, used in the definition of both $\gamma_{x y}$ and $\gamma_{c}$. This complicates the interpretation of the added broadband signal as a perturbation to the main signal. One can correct for this effect, however, by using $\operatorname{Re}\left\{\gamma_{c}\right\} /\left(1-\operatorname{Re}\left\{\gamma_{c}\right\}\right)$ instead of $\operatorname{Re}\left\{\gamma_{c}\right\}$. This correction is valid for any pair of signals with nonzero noise, which therefore have a coherence less than 1.0. The experimental measurements taken with this diagnostic have nonzero noise. 
To explain further, $\gamma_{c}$ represents, at each frequency, the fluctuating power normalized to the total signal power. Since the total signal power includes the fluctuating power, this normalization is not entirely appropriate when the fluctuating power makes us a significant fraction of the total power. Integrating $\operatorname{Re}\left\{\gamma_{c}\right\} /\left(1-\operatorname{Re}\left\{\gamma_{c}\right\}\right)$ accounts for the extra power in the denominator of the autospectral density function due to the fluctuations themselves. As the synthetic data shows, this integration is always superior to using $\operatorname{Re}\left\{\gamma_{c}\right\}$ alone, though it converges at large bandwidths or low fluctuation amplitudes.

In addition to the results shown here, common broadband fluctuation levels of 0 to $100 \%$ were tested using the same technique. The results at all finite fluctuation levels were similar to those shown in Figure2, with higher fluctuation levels only further favoring $\operatorname{Re}\left\{\gamma_{c}\right\} /\left(1-\operatorname{Re}\left\{\gamma_{c}\right\}\right)$ as the most accurate calculation method. With zero common signal, calculations using $\gamma_{x y}$ and $\gamma_{x y} /\left(1-\gamma_{x y}\right)$ still show finite fluctuation levels due to integration of noise, further emphasizing the possible complications with using these calculations. $\operatorname{Re}\left\{\gamma_{c}\right\}$ and $\operatorname{Re}\left\{\gamma_{c}\right\} /\left(1-\operatorname{Re}\left\{\gamma_{c}\right\}\right)$, on the other hand, show scatter near zero, as one would expect.

Taking both of these effects into account, Figure 2 shows that integrating $\operatorname{Re}\left\{\gamma_{c}\right\} /\left(1-\operatorname{Re}\left\{\gamma_{c}\right\}\right)$ most accurately recovers the true fluctuation level over the widest range of signal bandwidths. The improvement in accuracy over using just $\operatorname{Re}\left\{\gamma_{c}\right\}$ addresses aspect 2 of Equation 1 and the improvement over using $\gamma_{x y} /\left(1-\gamma_{x y}\right)$ addresses aspect 1 of Equation 1.

\section{Background Subtraction}

As discussed above, in addition to utilizing $\operatorname{Re}\left\{\gamma_{c}\right\} /\left(1-\operatorname{Re}\left\{\gamma_{c}\right\}\right)$ in the integrand of Equation 1. when working with real data one must also subtract a coherent background, $\gamma_{b g}$, from some pairs of CECE channels. This subsection will describe the reason for such subtraction as well as how this background subtraction affects the final results.

It was discovered early in the operation of the new diagnostic that different pairs of channels have considerably different background levels of $\gamma_{c}$. This background presents itself as a baseline level of the complex coherence function that is present at all frequencies. This background originates in finite filter overlap between some pairs of filters, but not others, since the bandpass functions are not entirely identical between all of the filters [11. This effect can be accurately assessed by injecting a noise source into the IF section input and calculating the coherence, thereby testing filter overlap without any plasma signal. For this purpose, the noise generated by the RF section amplifiers is sufficient to act as a test noise source. This testing can thus be performed regularly and shows the same common background level even with no plasma signal. See Ref. [11 for further discus-
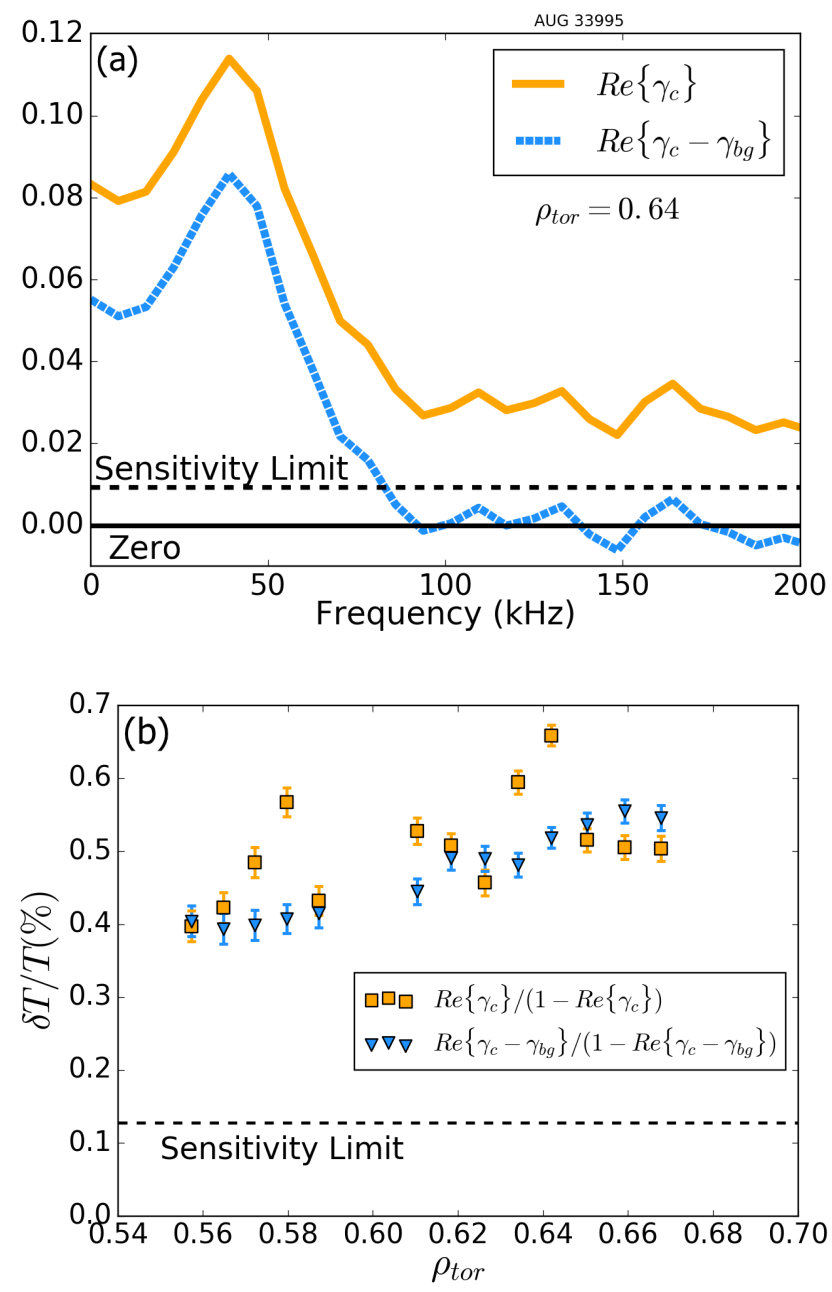

FIG. 3: Effect of background subtraction on experimental data for (a) spectra and (b) fluctuation profiles. Orange solid line in (a) and squares in (b) are without background subtraction. Blue dotted line in (a) and triangles in (b) are with background subtraction. Background subtraction effectively brings the spectrum to within the sensitivity limit at high frequency and removes unphysical structure from the profile. Error bars in (b) represent two standard deviations. $\rho_{\text {tor }}$ is defined as the square root of the normalized toroidal flux.

sion of finite filter overlap in CECE diagnostics, including example cross-power spectra.

This background manifests itself in plots of $\gamma_{c}$ against frequency, in which the spectrum never drops below the sensitivity limit, even in frequency ranges where there should be no coherent signal. See Figure 3 (a) for an example of an artificially raised level of $\gamma_{c}$ at high frequency. Non-plasma testing shows similar results, and reveals that this background is constant over the frequency ranges of interest (0 to $200 \mathrm{kHz}$ ). In addition, once the complex coherence function is integrated to obtain 
the total fluctuation level, the background artificially increases this total fluctuation level on some channel pairs. This artificial increase can create large discrepancies in the fluctuation level measured by channels that are quite close to one another, as seen in Figure 3 (b).

In order to resolve this issue of coherent noise between some channels but not others, the background level of $\gamma_{c}$, defined in Equation 4 as $\gamma_{b g}$, was subtracted off from each pair of channels before the integration. The appropriate level of background subtraction is determined by calculating the mean value of $\gamma_{c}$ at frequencies above those at which a measurable turbulent signal is present. In the examples here and in Section IV this background was calculated in the frequency range of 120 to $200 \mathrm{kHz}$.

Figure 3 (a) shows the effect of subtracting $\gamma_{b g}$ on an example frequency spectrum. The background subtraction brings the signal to within the sensitivity limit at high frequencies where there should be no measurable fluctuation signal. In addition, Figure 3 (b) shows the fluctuation profiles with and without background subtraction. The profile without background subtraction (orange squares) clearly has a large scatter in channels located close to each other. This profile appears to have unphysical structure, due solely to the differing backgrounds between different channel pairs. The use of background subtraction (blue triangles) most effectively reduces scatter and minimizes unphysical structure in the profile.

The data shown in Figure 3 addresses the third aspect of Equation 1, subtraction of $\gamma_{b g}$ from $\gamma_{c}$ before integration. Taking these results into account, the remainder of this paper will use Equation 1 to calculate all fluctuation levels from the CECE diagnostic.

\section{EXPERIMENTAL RESULTS}

The newly upgraded CECE IF section was installed on AUG in the Spring of 2017, and began taking data as part of the 2017 ASDEX Upgrade experimental campaign. As just one example of the diagnostic's improved capabilities, data was collected from a series of three repeat steady plasma discharges with the following parameters: $2.5 \mathrm{~T}$ field on axis, $1.0 \mathrm{MA}$ plasma current, $2.0 \cdot 10^{19} \mathrm{~m}^{-3}$ line averaged density, and 1.0 MW of electron cyclotron heating.

Figure 4 (a) shows the temperature fluctuation spectra from one of the discharges, plotted with $\gamma_{c}$ as defined in Equation 2 and $\gamma_{b g}$ as defined in Equation 4 . from three pairs of channels at radial locations of $\rho_{\text {tor }}=0.58$, 0.63 , and 0.66 , where $\rho_{\text {tor }}$ is the square root of the normalized toroidal flux. Uncertainty is represented by the shaded regions, and is calculated with Equation 5. The dotted black line shows two standard deviations above zero. This data was collected over 3 seconds of a steady L-mode plasma, during which all three radial locations were optically thick $(\tau>2)$.

All three channel pairs show measurable fluctuations,
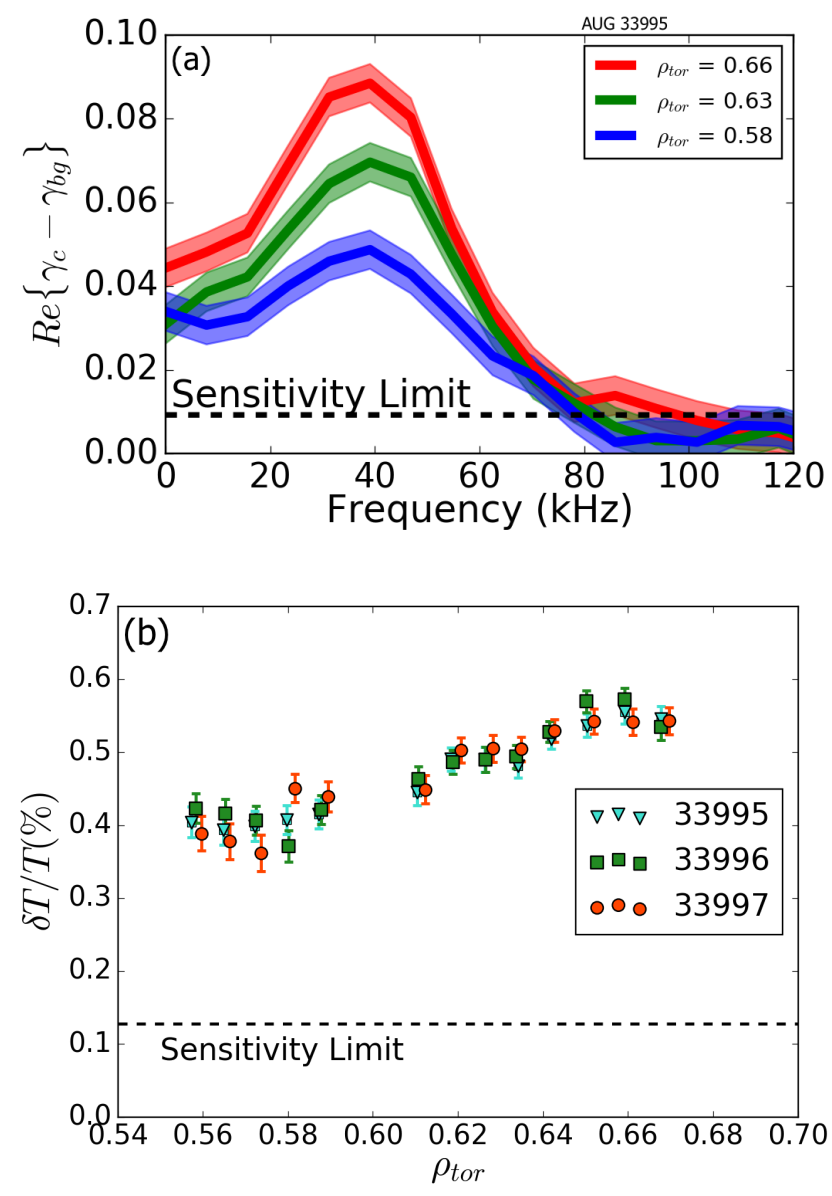

FIG. 4: Experimental measurements with the new CECE system on AUG. (a) Electron temperature fluctuation spectra after background subtraction, $R e\left\{\gamma_{c}-\gamma_{b g}\right\}$, from three radial locations in Shot 33995 . Uncertainty is represented by the shaded regions around each curve. (b) An example radial profile of temperature fluctuation levels from three repeat discharges. Fluctuations integrated from 20 to $100 \mathrm{kHz}$.

Error bars represent two standard deviations of uncertainty. $\rho_{\text {tor }}$ is defined as the square root of the normalized toroidal flux.

with $\widetilde{T} / T=0.41 \%, 0.50 \%$, and $0.57 \%$ (calculated using Equation 1 for $\rho_{\text {tor }}=0.58,0.63$, and 0.66 respectively. Fluctuations were integrated from 20 to $100 \mathrm{kHz}$ in order to capture the maximum fluctuation frequency above the sensitivity limit for the outermost channel, and to avoid known low frequency MHD activity. These are all above the sensitivity limit of $0.13 \%$, calculated with Equation 7 .

In a similar fashion, one can also calculate the integrated fluctuation level for each set of adjacent channels in order to construct a radial profile of the fluctuation levels. The fluctuation profiles for the three plasma discharges discussed above are shown in Figure 4 (b). The fluctuation levels here are calculated with Equation 1 . 
the uncertainty with Equation 6, and the sensitivity level with Equation 7. These profiles show that the fluctuation level rises nearly monotonically from approximately $0.4 \%$ to $0.6 \%$ over the radial range of $\rho_{\text {tor }}=0.56$ to $\rho_{\text {tor }}=0.68$. The increase in fluctuation level with increasing radius is consistent with past observations on other machines [5, 13.

Figure 4 (b) also shows that the profiles measured in all three shots generally agree within the uncertainty of the diagnostic, indicating that the system is able to make highly repeatable measurements in similar plasma conditions.

In addition to the discharges shown here, the hardware and analysis techniques described here have been successfully applied to more than 20 other discharges in order to measure electron temperature fluctuations.

The data shown in Figure 4 testifies to the significantly expanded capabilities of the new CECE system. First, the ability to measure a wide radial profile in a single plasma discharge is a major advantage of the new system, since previous CECE systems often required a series of repeat discharges in order to measure radial profiles with more than a few points [13]. This is made possible by the large number of channels and their comb-like spacing. In addition, the ability to make reliable measurements deep into the core is only possible due to the reduced electronics noise of the new system.

\section{CONCLUSIONS}

A new correlation electron cyclotron emission (CECE) intermediate frequency section has been installed on ASDEX Upgrade and has successfully begun taking data in the 2017 experimental campaign. The new system has reduced electronics noise and 30 channels, enabling finely resolved radial profile measurements of temperature fluctuations, as well as measurement of radial correlation lengths in the same discharge, which are to be completed. In addition, significantly improved statistical analysis techniques have been developed to analyze the raw CECE data, and are both derived here and tested with synthetic data. Future work will include coupled CECE and reflectometer measurements and possibly new dedicated optics.

\section{ACKNOWLEDGEMENTS}

This work has been carried out within the framework of the EUROfusion Consortium and has received funding from the Euratom research and training programme 2014-2018 under grant agreement No 633053. The views and opinions expressed herein do not necessarily reflect those of the European Commission. This work is also supported by the US DOE under Grants DE-SC0006419 and DE-SC0017381. In addition, this research is supported by the US DoD and the Air Force Office of
Scientific Research under the National Defense Science and Engineering Graduate (NDSEG) Fellowship, 32 CFR $168 \mathrm{a}$.

\section{Appendix A: Frequency Domain Derivation of Temperature Fluctuation Calculation}

This section will derive Equation 1 using the definition of $\gamma_{c}$ from Equation 2, highlighting differences with previous derivations that have led to Equation 9 .

Consider one signal collected by an ECE radiometer, $x(t)$, which includes contributions from the steady state temperature $T_{e}$, temperature fluctuations $\widetilde{\mathcal{T}}_{e}(t)$, and thermal noise $\tilde{\mathcal{N}}_{x}(t)$ [8, 29].

$x(t)=c_{x}\left(T_{e}+\widetilde{\mathcal{T}}_{e}(t)\right)\left(1+\tilde{\mathcal{N}}_{x}(t)\right) \approx c_{x}\left(T_{e}+\widetilde{\mathcal{T}}_{e}(t)+T_{e} \tilde{\mathcal{N}}_{x}(t)\right)$

where $c_{x}$ is a calibration factor that relates the radiometer signal to the absolute temperature. This factor will cancel out later in this derivation, so absolute calibration is not necessary for CECE operation using this calculation method. It is assumed in this derivation that $\widetilde{\mathcal{T}}_{e} \ll T_{e}$ and $\widetilde{\mathcal{N}}_{x} \ll 1$, so that the quadratic term $\widetilde{\mathcal{T}}_{e} \widetilde{\mathcal{N}}_{x}$ is negligibly small [8].

One then takes the Fourier transform of Equation A1, defining $X(f)$ to be the Fourier transform of $x(t), \widetilde{T}_{e}(f)$ to be the Fourier transform of $\widetilde{\mathcal{T}}_{e}(t)$, and $\widetilde{N}_{x}(f)$ to be the Fourier transform of $\widetilde{\mathcal{N}}_{x}(t)$. This gives:

$$
X(f)=c_{x}\left(\delta(f) T_{e}+\widetilde{T}_{e}(f)+T_{e} \widetilde{N}_{x}(f)\right)
$$

where $\delta(f)$ is the Dirac Delta Function, indicating that the background temperature leads to a constant component at zero frequency.

This derivation is concerned with only the fluctuating part of the frequency spectrum $(f \neq 0)$ such that one can ignore the equilibrium temperature. Consider now the cross-spectral density function [20] of two signals $x$ and $y$ :

$$
G_{x y}(f)=\left\langle X(f)^{*} Y(f)\right\rangle
$$

where the triangle brackets represent ensemble averaging and the asterisk represents the complex conjugate, and the auto-spectral density function of $x$ :

$$
G_{x x}(f)=\left\langle X(f)^{*} X(f)\right\rangle
$$

Inserting Equation A2 into Equation A3 gives:

$$
\begin{aligned}
G_{x y}(f)= & c_{x} c_{y}\left(\left\langle\widetilde{T}_{e}^{*}(f) \widetilde{T}_{e}(f)\right\rangle+T_{e}\left\langle\widetilde{N}_{x}^{*}(f) \widetilde{T}_{e}(f)\right\rangle\right. \\
& \left.+T_{e}\left\langle\widetilde{T}_{e}^{*}(f) \tilde{N}_{y}(f)\right\rangle+\left\langle T_{e}^{2} \widetilde{N}_{x}^{*}(f) \widetilde{N}_{y}(f)\right\rangle\right)
\end{aligned}
$$


Since the thermal noise in each channel is uncorrelated with the turbulent temperature fluctuations, the $\langle\widetilde{T} \widetilde{N}\rangle$ terms are dropped, as in References [8, 10] and others (even if correlated, these terms would be quadratic in small parameters). Note that ensemble averaging brackets will be dropped in the remainder of this derivation to minimize notational clutter. This then reduces to:

$$
\begin{aligned}
G_{x y}(f) & =c_{x} c_{y}\left(\widetilde{T}_{e}^{2}(f)+T_{e}^{2} \widetilde{N}_{x y}^{2}\right) \\
& =c_{x} c_{y} \widetilde{T}_{e}^{2}(f)+G_{n o i s e}
\end{aligned}
$$

This assumes that the thermal noises $\widetilde{N}_{x}$ and $\widetilde{N}_{y}$ are mostly uncorrelated, except for some small part $\tilde{N}_{x y}$ caused, for example, by finite filter overlap. $G_{\text {noise }}=$ $c_{x} c_{y} T_{e}^{2} \widetilde{N}_{x y}^{2}$ is the portion of the cross-spectral density due to common noise between the two channels. As described below, this makes this derivation more general than most previous derivations that assume $\widetilde{N}_{x y}^{2}=0$.

Similarly,

$$
G_{x x}(f)=c_{x}^{2}\left(\widetilde{T}_{e}^{2}(f)+T_{e}^{2} \widetilde{N}_{x}^{2}(f)\right)
$$

Note that this step differs from pervious derivations in two ways, both of which make the current derivation more generally applicable than previous derivations (for example, in Reference [31]). First, most previous derivations have assumed $\widetilde{T}_{e}^{2} \ll T_{e}^{2} \widetilde{N}_{x}^{2}$, such that $G_{x x}(f) \approx c_{x}^{2}\left(T_{e}^{2} \widetilde{N}_{x}^{2}\right)$ (note that the definitions of $c_{x}$ and $\widetilde{N}_{x}^{2}$ here are slightly different than in Reference [31]). Relaxing this assumption leads to the eventual integrand of $\gamma_{c} /\left(1-\gamma_{c}\right)$ in Equation 1 as opposed to just $\gamma_{c}$ (aspect 2 from Section IIIA).

In addition, this derivation allows for some common noise between channels $x$ and $y$ by keeping a term $G_{n o i s e}$, whereas previous derivations have assumed this term to be identically zero (this will later prove to be related to aspect 3 from Section III A Keeping this additional term is an important extension over previous derivations.

Solving Equation A7 for $c_{x}$ gives:

$$
c_{x}=\sqrt{\frac{G_{x x}(f)}{\widetilde{T}_{e}^{2}(f)+T_{e}^{2} \widetilde{N}_{f}^{2}}}
$$

This assumes that the magnitude of the thermal noise on both channels is the same, even if the noise between the two channels is uncorrelated $\left(\widetilde{N}_{x}^{2}(f) \approx \widetilde{N}_{y}^{2}(f) \approx \widetilde{N}_{f}^{2}\right.$, where the $f$ subscript emphasizes that this is noise per unit frequency). This assumption should be valid in any case where all CECE assumptions are valid (the two channels are closely enough spaced that they are sampling roughly the same background temperature). Such an assumption is also later justified by Equation A16.

Now, solve Equation A6 for $\widetilde{T}_{e}^{2}(f)$ :

$$
\widetilde{T}_{e}^{2}(f)=\frac{G_{x y}(f)-G_{n o i s e}}{c_{x} c_{y}}
$$

Then insert Equation $\mathrm{A} 8$ and the equivalent for $c_{y}$ to get:

$$
\widetilde{T}_{e}^{2}(f)=\frac{G_{x y}(f)-G_{n o i s e}}{\sqrt{G_{x x}(f) G_{y y}(f)}}\left(\widetilde{T}_{e}^{2}(f)+T_{e}^{2} \widetilde{N}_{f}^{2}\right)
$$

One can then define the complex coherence function, $\gamma_{c}$, (see page 390 of Ref. [19]) as is done in Equation 2. For the sake of clarity it is repeated here:

$$
\gamma_{c}(f)=\frac{G_{x y}(f)}{\sqrt{G_{x x}(f) G_{y y}(f)}}
$$

In addition, Equation 4 will be written in terms of $G_{n o i s e}, G_{x x}$, and $G_{y y}$ :

$$
\gamma_{b g}=\frac{\overline{G_{n o i s e}}}{\sqrt{\overline{G_{x x}} \overline{G_{y y}}}}
$$

where the overbar represents a frequency average over a frequency range far above the turbulent signal (assuming a constant background over the frequency range of interest, which is the case in all of the experimental discharges considered in this study). The value of $\gamma_{b g}$ is calculated experimentally using Equation 4.

Using these definitions, one can write:

$$
\widetilde{T}_{e}^{2}(f)=\left(\gamma_{c}(f)-\gamma_{b g}\right)\left(\widetilde{T}_{e}^{2}(f)+T_{e}^{2} \widetilde{N}_{f}^{2}\right)
$$

Rearranging, solving for $\widetilde{T}_{e}^{2}(f)$, and dividing by $T_{e}^{2}$ gives:

$$
\frac{\widetilde{T}_{e}^{2}(f)}{T_{e}^{2}}=\tilde{N}_{f}^{2} \frac{\gamma_{c}(f)-\gamma_{b g}}{1-\left(\gamma_{c}(f)-\gamma_{b g}\right)}
$$

Integrating over the frequency range of interest and considering the root-mean-square value of the temperature fluctuation level (as in Reference [8] and others) gives:

$$
\left.\frac{\widetilde{T}}{T}\right|_{r m s}=\sqrt{\int_{f_{1}}^{f_{2}} \tilde{N}_{f}^{2} \frac{R e\left\{\gamma_{c}(f)-\gamma_{b g}\right\}}{1-\operatorname{Re}\left\{\gamma_{c}(f)-\gamma_{b g}\right\}} d f}
$$

where one takes the real part of the complex coherence function in order to obtain the root-mean-square value of the temperature fluctuations [20]. Note that this integral gives the band-limited turbulent temperature fluctuations from frequency $f_{1}$ to $f_{2}$, which, as stated in the 
main text, are determined by the frequency width of the turbulent feature.

For a finite frequency bandwidth $\left(f_{1}\right.$ to $\left.f_{2}\right)$ one can substitute an expression for the thermal noise. From References [29, 32, 33, for an ECE radiometer the measured fluctuation level due to thermal noise is:

$$
\left.\frac{\widetilde{T}_{e}^{2}}{T_{e}^{2}}\right|_{\text {noise }}=\int_{f_{1}}^{f_{2}} \widetilde{N}_{f}^{2} d f=B_{\text {sig }} \widetilde{N}_{f}^{2}=\frac{2 B_{\text {sig }}}{B_{I F}}
$$

where $B_{I F}$ is the intermediate frequency bandwidth and $B_{s i g}=f_{2}-f_{1}$ is the signal bandwidth (allowing the signal bandwidth $B_{\text {sig }}$ to be less than the video bandwidth). Thus, $\widetilde{N}_{f}^{2}=2 / B_{I F}$. If possible, it is also advantageous to measure $\widetilde{N}_{f}^{2}$ experimentally, rather than using the theoretical value.

Inserting this into Equation A15 gives the desired result, Equation 1 .

$$
\left.\frac{\widetilde{T}}{T}\right|_{r m s}=\sqrt{\frac{2}{B_{I F}} \int_{f_{1}}^{f_{2}} \frac{\operatorname{Re}\left\{\gamma_{c}(f)-\gamma_{b g}\right\}}{1-\operatorname{Re}\left\{\gamma_{c}(f)-\gamma_{b g}\right\}} d f}
$$

Note that in Equation 1 the $\left.\right|_{r m s}$ notation has been dropped for the sake of brevity.

Most previous derivations of an equation for the total temperature fluctuation level from a CECE diagnostic have proceeded in the time domain, rather than the frequency domain [8, 29, 31]. Though the derivation presented in this Appendix was performed in the frequency domain, as the authors believe this to be clearer and more concise, it is also possible to perform the same derivation, starting with Equation A1 in a manner more similar to past work. Instead of Fourier transforming immediately, one defines the autocorrelation and crosscorrelation functions as [20]:

$$
R_{i j}(\tau)=\frac{1}{t_{\text {tot }}} \int_{0}^{t_{t o t}} i(t) j(t+\tau) d t
$$

Inserting the time domain signals gives equations that are the time domain analogues of Equations A6 and A7. One then solves for the constants $c_{x}$ and $c_{y}$ in the same manner as in the derivation presented here, combines equations, and rearranges to get the analogue of Equation A10, except that it contains correlation functions rather than spectral density functions:

$$
\frac{\widetilde{T}_{e}^{2}}{T_{e}^{2}}=\widetilde{\mathcal{N}}^{2} \frac{\frac{R_{x y}(0)}{\sqrt{R_{x x}(0) R_{y y}(0)}}-\frac{R_{\text {noise }}}{\sqrt{R_{x x}(0) R_{y y}(0)}}}{1-\left(\frac{R_{x y}(0)}{\sqrt{R_{x x}(0) R_{y y}(0)}}-\frac{R_{n o i s e}}{\sqrt{R_{x x}(0) R_{y y}(0)}}\right)}
$$

Note that the $\widetilde{\mathcal{N}}^{2}$ in this equation is different than the $\widetilde{N}_{f}^{2}$ from Equation A10 (the former is a total noise level while the latter is the noise level per unit frequency).

One then uses the relationship between correlation functions and spectral density functions in general (they are Fourier transforms on one another [20]), and specifically the realtionship [34]:

$$
\frac{R_{x y}(0)}{\sqrt{R_{x x}(0) R_{y y}(0)}}=\frac{1}{B_{s i g}} \int_{f_{1}}^{f_{2}} \operatorname{Re}\left\{\gamma_{c}(f)\right\} d f
$$

where $B_{s i g}$ and $\gamma_{c}$ are defined as above. This relationship is exactly true in the limit of a continuous integral 34. Inserting this relationship (and the equivalent for the noise term) into Equation A19 and using $\widetilde{\mathcal{N}}^{2}=B_{\text {sig }} \widetilde{N}_{f}^{2}=2 B_{\text {sig }} / B_{I F}$ gives the desired result, which is the same as in the frequency domain derivation (the same as Equation A17):

$$
\frac{\widetilde{T}}{T}=\sqrt{\frac{2}{B_{I F}} \int_{f_{1}}^{f_{2}} \frac{\operatorname{Re}\left\{\gamma_{c}(f)-\gamma_{b g}\right\}}{1-\operatorname{Re}\left\{\gamma_{c}(f)-\gamma_{b g}\right\}} d f}
$$

[1] W. Horton, Rev. Mod. Phys. 71 (3), 735 (1999).

[2] C. Holland, Phys. Plasmas 23, 060901 (2016).

[3] C. Holland, A. E. White, G. R. McKee, M. W. Schafer, J. Candy, R. E. Waltz, L. Shmitz, and G. Tynan, Phys. Plasmas 16, 052301 (2009).

[4] A. E. White, N. T. Howard, A. J. Creely, M. A. Chilenski, M. Greenwald, A. E. Hubbard, J. W. Hughes, E. Marmar, J. E. Rice, J. M. Sierchio, C. Sung, J. R. Walk, D. G. Whyte, D. R. Mikkelsen, E. M. Edlund, C. Kung, C. Holland, J. Candy, C. C. Petty, M. L. Reinke, and C. Theiler, Phys. Plasmas 22, 056109 (2015).

[5] A. J. Creely, N. T. Howard, P. Rodriguez-Fernandez, N. Cao, A. E. Hubbard, J. W. Hughes, J. E. Rice, A. E.
White, J. Candy, G. M. Staebler, G. D. Conway, S. J. Freethy, and C. Sung, Phys. Plasmas 24, 056104 (2017).

[6] S. Sattler and H. J. Hartfuss, Phys. Rev. Lett. 72, 653 (1993).

[7] C. Watts, R. F. Gandy, T. D. Rempel, and G. Cima, Rev. Sci. Instrum. 66, 451 (1995).

[8] G. Cima, R. V. Bravenec, A. J. Wootton, T. D. Rempel, R. F. Gandy, C. Watts, and M. Kwon, Phys. Plasmas 2, 720 (1995).

[9] C. Watts, Y. In, J. Heard, P. Phillips, A. Lynn, A. Hubbard, and R. Gandy, Nucl. Fusion 44 (9), 987 (2004).

[10] C. Watts, Fusion Sci. Technol. 52, 176 (2007).

[11] A. E. White, L. Schmitz, W. A. Peebles, T. A. Carter, 
T. L. Rhodes, E. J. Doyle, P. A. Gourdain, J. C. Hillesheim, G. Wang, C. Holland, G. R. Tynan, M. E. Austin, G. R. McKee, M. W. Shafer, K. H. Burrell, J. Candy, J. C. DeBoo, R. Prater, G. M. Staebler, R. E. Waltz, and M. A. Makowski, Rev. Sci. Instrum. 79 (10), 103505 (2008).

[12] C. Sung, A. E. White, J. H. Irby, R. Leccacorvi, R. Vieira, C. Y. Oi, W. A. Peebles, and X. Nguyen, Rev. Sci. Instrum. 83, 10E311 (2012).

[13] N. T. Howard, C. Sung, and A. E. White, Rev. Sci. Instrum. 85, 11D811 (2014).

[14] S. E. Zemedkun, S. Che, Y. Chen, C. W. Domier, N. C. L. Jr., T. Munsat, S. E. Parker, B. Tobias, W. Wan, and L. Yu, Phys. Plasmas 22, 122508 (2015).

[15] S. J. Freethy, G. D. Conway, I. Classen, A. J. Creely, T. Happel, A. Koehn, B. Vanovac, and A. E. White, Rev. Sci. Instrum. 87, 11E102 (2016).

[16] C. Sung, W. A. Peebles, C. Wannberg, T. L. Rhodes, X. Nguyen, R. Lantsov, and L. Bardczi, Rev. Sci. Instrum. 87, 11E123 (2016).

[17] M. Fontana, L. Porte, and P. M. Cabrera, Rev. Sci. Instrum. 88, 083506 (2017).

[18] T. Happel, G. D. Conway, W. Kasparek, B. Plaum, C. Lechte, D. Wagner, U. Stroth, and the ASDEX Upgrade Team, in Proceedings of the 10th International Reflectometry Workshop (2011) pp. 1-6.

[19] S. L. Marple, Digital Spectral Analysis: With Applications (Prentice Hall, 1987).

[20] J. S. Bendat and A. G. Piersol, Random Data, 2nd ed. (Wiley, 2000).

[21] D. E. Smith, E. J. Powers, and G. S. Caldwell, IEEE Transactions on Plasma Science PS-2, 261 (1974).
[22] N. Weiner, Acta Mathematica 55, 117 (1930).

[23] G. C. Carter and C. H. Knapp, IEEE Transactions on Acoustics, Speech, and Signal Processing ASSP-23, 257 (1975).

[24] K. D. Saunders and F. C. Hanrick, Journal of Geophysical Research 87, 9699 (1982).

[25] G. C. Carter, Proceedings of the IEEE 75, 236 (1987).

[26] W. H. . Carter, in Handbook of Optics: Volume I: Fundamentals, Techniques, and Design, edited by M. Bass, E. W. V. Stryland, D. R. Williams, and W. L. Wolfe (McGraw-Hill, Inc., 1995) Chap. 4, p. 4.12.

[27] P. Stoica and R. Moses, Spectral Analysis of Signals (Prentice Hall, 2002).

[28] R. Shiavi, Introduction to Applied Statistical Signal Analysis (Elsevier Inc., 2007).

[29] S. Sattler and H. J. Hartfuss, Plasma Phys. Control. Fusion 35, 1285 (1993).

[30] V. S. Udintsev, M. Goniche, J. L. Segui, G. Giruzzi, D. Molina, F. Turco, G. T. A. Huysmans, P. Maget, T. S. Team, and A. Kraemer-Flecken, Fusion Sci. Technol. 50, 508 (2006).

[31] C. Sung, A. E. White, N. T. Howard, C. Y. Oi, J. E. Rice, C. Gao, P. Ennever, M. Porkolab, F. Parra, D. Mikkelsen, D. Ernst, J. Walk, J. Hughes, J. Irby, C. Kasten, A. E. Hubbard, M. J. Greenwald, and the Alcator CMod Team, Nucl. Fusion 53, 083010 (2013).

[32] M. Tiuri, IEEE Transactions on Military Electronics 8, 264 (1964).

[33] G. Bekefi, Radiation Processes in Plasmas (Wiley, 1966).

[34] W. A. Gardner, Signal Processing 29, 113 (1992). 